\title{
Learning from Crickets: Artificial Hair-Sensor Array Developments
}

\author{
Gijs J.M. Krijnen, Theo Lammerink and R. Wiegerink \\ Transducers Science \& Technology group \\ $\mathrm{MESA}^{+}$Research Institute for Nanotechnology, University of Twente \\ Enschede, The Netherlands \\ gijs.krijnen@utwente.nl
}

\begin{abstract}
We have successfully developed biomimetic flowsensitive hair-sensor arrays taking inspiration from mechanosensory hairs of crickets. Our current generation of sensors achieves sub $\mathrm{mm} / \mathrm{s}$ threshold air-flow sensitivity for single hairs operating in a bandwidth of a few hundred $\mathrm{Hz}$ and is the result of a few iterations in which the natural system (i.e. crickets filiform hair based mechano-sensors) have shown ample guidance to optimization. Important clues with respect to mechanical design, aerodynamics, viscous coupling effects and canopy based signal processing have been used during the course of our research. It is only by consideration of all these effects that we now may start thinking of systems performing a "flow-camera" function as found in nature in a variety of species.
\end{abstract}

\section{INTRODUCTION}

\section{A. Flow as information source}

Whereas for humans (mammals) using flow may not often seem a very information rich modality for probing the environment, nature seems to have numerous examples of species that exactly do this. To put this in perspective it may be helpful to look at the fields produced by a harmonically moving sphere (dipole), which, with some simplifications, may resemble more natural sources such as wing-beats of flying insects or tail movements of fish. Obviously the fields produced by a dipole entail both pressure and flow fields. Depending on the medium, e.g. water or air, in which the dipole resides, and more specifically on the mediums compressibility, it may seem that pressure and flow could play comparably important roles, albeit that flow-fields inherently carry directional information (being vector-fields) whereas pressure is a scalar field only. How much this observation may be true at relatively long distances (for compressible media one could think of a few wavelengths away from the source) where the ratio of flow- and pressure fields becomes constant, near to the source flow-fields are comparatively "more present". This is shown in Fig. 1 where on-axis pressure and velocity, normalized to their respective values at $k \cdot r=1$ (with $k$ the wavenumber and $r$ the distance), are plotted as a function

This research was made possible by grants from the Customized Intelligent Life-Inspired Arrays project funded by the Future and Emergent Technologies arm of the EU IST Programme and by the BioEARS Vici grant of the Dutch Technology Foundation (STW/NWO).

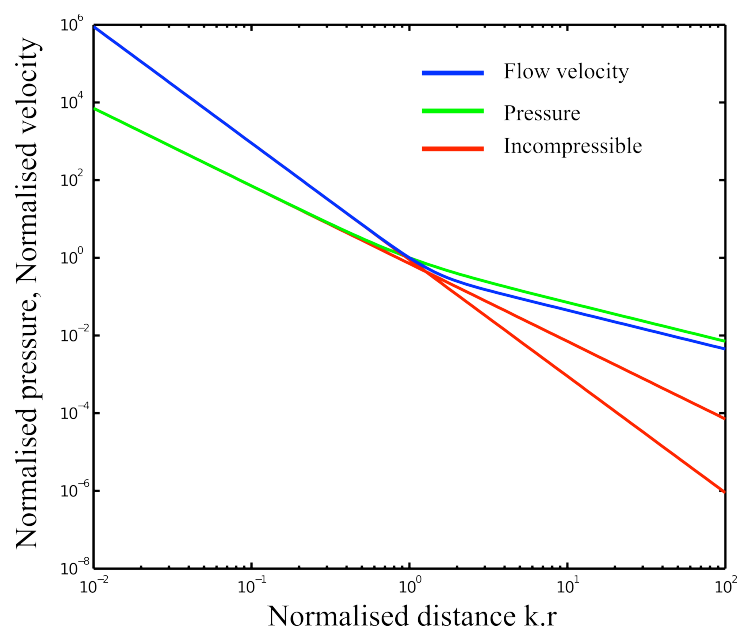

Figure 1. Dipole source pressure and velocity normalised to their respective values at $k \cdot r=1$. Red lines indicate incompressible fluids.

of distance. Both the situations of compressible (green, blue) and incompressible (red) media, as calculated from the expressions in [1], are shown. Clearly, for $k \cdot r=1$, i.e. distances small compared to the wavelength, there are only minor differences between compressible and incompressible theory, a fact that can be readily exploited when modeling relative complex aerodynamic predator-prey interactions [2].

The ratio between pressure and flow velocity (which would be the acoustic impedance for compressible media) equals $j \rho \omega r / 2$ ( $\rho$ being the density, $\omega$ the angular frequency) for $k \cdot r<<1$ indicating that pressure becomes comparatively small for shorter distances and lower frequencies. As an example for an interaction of a flying wasp with a wing beat of $\approx 150 \mathrm{~Hz}$ with, say, a caterpillar [3] the condition $k \cdot r=1$ corresponds with $r \approx 0.34 \mathrm{~m}$ a relative large distance as measured in body lengths of the animals.

\section{B. Hairs as mechanical transducers}

Like membranes are often found in sensory systems capturing pressure differences, e.g. as seen in the ears of 


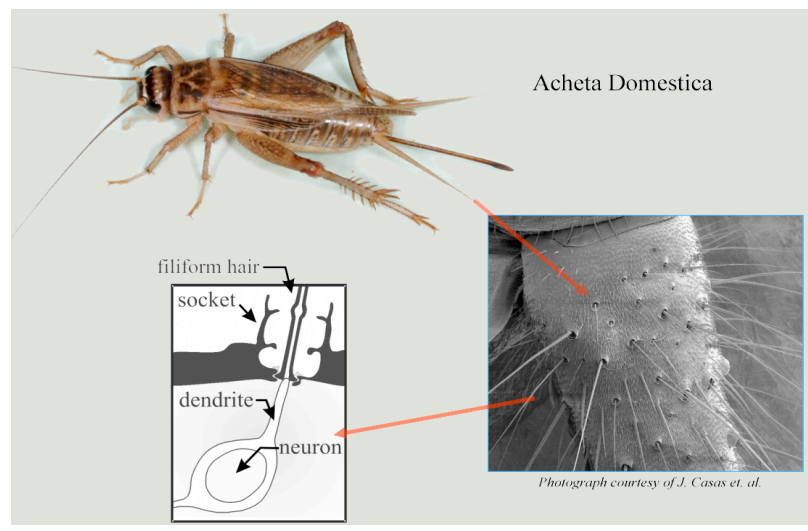

Figure 2. Flow-sensitive hair-sensors are found, amongst others, on cerci, the conical appendages found at the abdominal side of crickets.

insects [4] or in impedance transformers found in the basilar membrane of the mammalian hearing system [5], nature seems to have a preference to pick up flow-information by hairs. Mechano-sensory hairs are abundantly found in arthropods for a variety of purposes, e.g. tactile sensing [6], gravity sensing, gyroscopic sensing, as well as sensing media motion. E.g. crickets are well known for their ability to perceive minute airdisplacements down to flow amplitudes of about $30 \mu \mathrm{m} / \mathrm{s}$, bordering at thermal-mechanical noise thresholds [7].

The hair-sensors for flow-sensing generally consist of rather thin hairs with varying length, mounted in a flexible socket allowing for drag-torque induced rotations. These rotations on their turn cause a mechanical strain on a receptor cell leading to the generation of an action potential (or the variation of the spike-rate of a continuously spiking cell). The hairs normally rotate most easily in a preferential direction due to an asymmetry in the socket. The rotational stiffness ratio between "easy" and "hard" direction of rotation varies between 4-fold and 8-fold [8]. This leads to a figure of eight directivity which is helpful in determining flow-direction [9]

\section{Cricket cerci as example system}

In arranging many hair-sensors into a canopy (array) such as has evolved in the form of the cerci found at the abdominal side of crickets, they have acquired the ability to accurately pinpoint flow directions down to $4-7$ degrees accuracy in the horizontal plane [10]. This greatly helps in detecting and localizing approaching predators, such as spiders, and, hence, the cercal sensory system is thought to play an important role in the cricket escape system. In fact the cercal system helps the crickets to "see their aerodynamic" world around them, albeit that this is so only for the aerodynamic near field environment. A system comparable in function is the fish lateral line system which allows fish to observe the hydrodynamic environment by a large number of superficial and canal-embedded flow-sensors (called neuromast) [11] in a space roughly scaled by the body-length of the fish [12]

We have used the cricket cercal sensory system as a source of inspiration. Using artificial hairs for drag-torque pick up, sitting on torsion beams, hair-rotation is used to modulate two capacitors differentially. The capacitors are read-out using charge amplifiers. For optimization of individual air-flow sensors as well as for obtaining clues on how to design arrays consisting of many sensors we have looked at various aspects of the cricket cercal system and incorporated the obtained insights in a number of design- and implementation iterations.

\section{SENSOR PRINCIPLE}

On a basal level the simple principle of a hair sitting on a torsional mount allowing for drag-induced rotation has been the starting point for our artificial hair sensors (see Fig. 3). In the implementation the hairs are made of SU-8 [13]. Using two deposition-exposure cycles and subsequent development these hairs can be made up to $1 \mathrm{~mm}$ long with diameters ranging between 25 and $50 \mu \mathrm{m}$. The rotational freedom comes from two torsion beams. These beams as well as two membranes connected to the beams are made of siliconnitride. On the membranes a metal layer $(\mathrm{Cr}$ in the early implementations and $\mathrm{Al}$ in the later ones) is deposited which forms a capacitor together with the underlying highly doped silicon substrate. The hairs are sitting on the membranes as well. Rotational freedom is obtained when the sacrificial polysilicon layer $(0.5-1.0 \mu \mathrm{m}$ thick $)$, which sits between the membranes and a protective silicon-nitride layer on the silicon substrate, is selectively removed (e.g. by $\mathrm{XeF}_{2}$-etching).

\section{OPTIMIZATION OF SINGLE HAIR SENSORS}

The conditions under which the hair-sensors have to operate are such that the Reynolds $(R)$ and Strouhal $(S t)$ numbers are relatively low. For a hair-diameter of $25-50 \mu \mathrm{m}$, an air-oscillation frequency of $250 \mathrm{~Hz}$ and a flow-velocity amplitude of $10 \mathrm{~mm} / \mathrm{s}, R$ varies between $0.008-0.016$ and $\mathrm{St}$ between 1.96 - 3.92 (for the flow around the hairs). The rather small Reynolds numbers and the large hair-length to hairdiameter ratio allow using the Stokes expressions for the dragtorque exerted by the air-flow on the hairs [14]. Further our hair-sensors are mounted on flat substrates allowing the use of the Stokes expressions for a harmonic flow along the hairs [15]. These expressions predict a viscous flow over an infinite substrate to be harmonic in time with zero flow-velocity and 45 degrees phase advance at the substrate interface and a boundary layer thickness $\left(\delta_{b}\right)$ proportional to the inverse of $\beta=(\omega / 2 v)^{0.5}$ where $v$ is the kinematic viscosity $\left(1.79 \cdot 10^{-5}\right.$ $\mathrm{m}^{2} / \mathrm{s}$ for air at room-temperature) and $\omega$ is the radial frequency. We have shown previously that the Stokes expressions can be usefully employed for our situation [16]. As an example, for a harmonic air-flow of $100 \mathrm{~Hz}$ the boundary layer is roughly $0.5 \mathrm{~mm}$. Note that under most conditions the artificial hairs can be assumed infinitely stiff

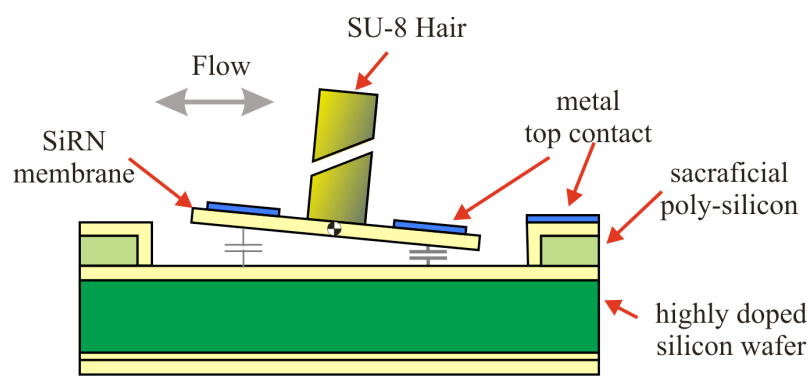

Figure 3: Schematic of artificial hair-based flow-sensor with differential capacitive lay-out. 


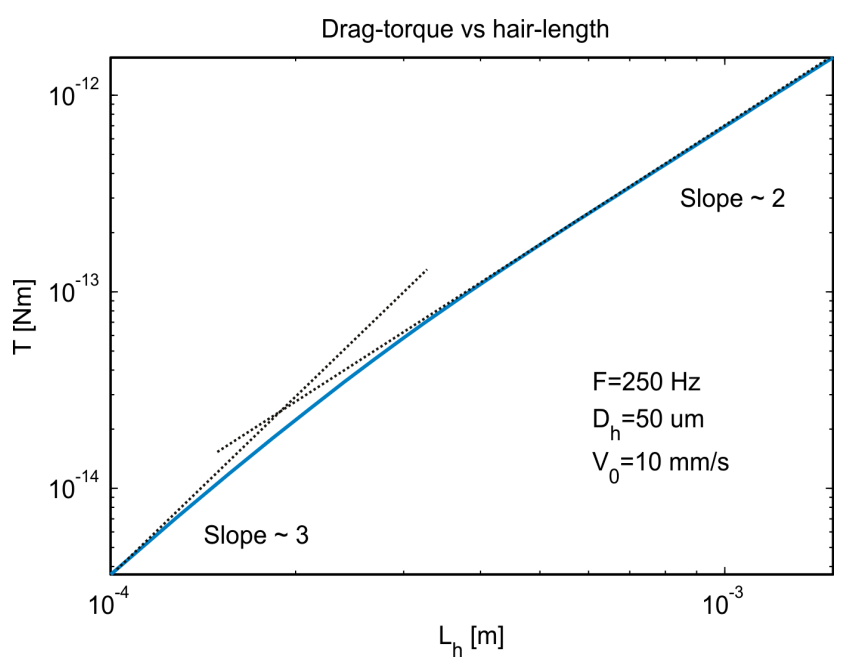

Figure 4: Flow-induced drag-torque as a function of hair-length.

and the rotation angles are rather small (in the order of $1-100$ mrad amplitude per $\mathrm{m} / \mathrm{s}$ flow-velocity amplitude).

\section{A. Hair length and boundary layer}

The length of the hairs $(L)$ plays a dominant role in the overall performance of the hair-sensors. Obviously, when exposed to a uniform flow the total drag torque on cylindrical hairs would increase proportional with the hair-length squared. However, due to the frequency depending boundary layer the drag-torque first increases with the third power of hair-length up to about $\delta_{b}$ and then follows a quadratic dependence. See Fig. 4. Moreover, the hair inertial moment $(I)$ is of order $O(L)^{3}$.

\section{B. Hair diameter and viscous drag}

When increasing the diameter of the hairs $(d)$ the resulting drag-force will increase as well. However, by evaluating the Stokes expressions for drag-force it turns out that the dependence on diameter is weak, on the order of $O(d)^{1 / 3}$. At the same time the hair moment of inertia increase is of order $O(d)^{2}$ affecting the resonance frequency negatively. Therefore it is beneficial to have thin hairs. Technologically it seems to be rather difficult to make hairs with aspect ratios of more than about $10-20$. We have tackled this problem by segmenting our artificial hairs with lower part diameter of $50 \mu \mathrm{m}$ and top part diameter of $25 \mu \mathrm{m}$ reducing the hair inertial moment by about $65 \%$ [17].

\section{Torsional stiffness}

Obviously, when looking for the largest rotation angle for any given drag-torque one may want to choose the lowest possible torsional stiffness $(S)$. However, for given hair inertial moment a reduction of $\mathrm{S}$ also leads to a reduction of the resonance frequency which is given by $\omega_{0}=(S / I)^{0.5}$.

\section{Damping}

Damping of the hair-sensors comes in multiple forms. For the crickets the hair-sockets provide some torsional damping
( $R$ ) by visco-elastic material properties (see [18] for such material properties in spider hair mechano sensors) whereas for the artificial hair-sensors torsional damping is caused by both material as well as squeeze film damping due to the small gap between the silicon-nitride plates and the substrate. On top of these damping contributions the hairs themselves incur damping by viscous forces when the hairs move relatively to the surrounding air. In the case of crickets the total damping seems to be appropriately controlled by the organism yielding hairs that are approximately critically damped. It is hypothesized that this mechanical impedance matching helps the sensors to obtain maximum energy from the surroundings [19]. On the other on critically damping a second order system one also maximizes its agility [20] and the overall reasons determining the appropriate damping for cricket hair-sensors have not yet been identified. In the artificial hair-sensors, except for adding specific holes to the membranes to tailor the squeeze film damping, not much can be done to optimize the damping without requiring far-reaching consequences for the fabrication technology.

\section{E. Material choices}

The mechanical sensitivity of our hair-sensors is currently about 2 orders of magnitude less than those of crickets, primarily due to a much larger rotational stiffness: $1.5 \cdot 10^{-11}$ $\mathrm{Nm} / \mathrm{rad}$ for crickets versus $4.85 \cdot 10^{-9} \mathrm{Nm} / \mathrm{rad}$ for our sensors. However, reducing the torsional stiffness comes with two difficulties. In order to conserve bandwidth the moment of inertia of the hairs needs to be further reduced. Therefore we are currently investigating fabrication of more nature-like hairs on which we will report in the near future [21]. The second complication is that the suspension beams provide torsional- as well as vertical compliance. The ratio of vertical compliance $K$ over rotational compliance $S$ at given desired value $S_{0}$, which ideally should be infinite, follows from combining the expressions for beambending

$$
K=2 E \cdot \frac{I_{x}}{L^{3}} \quad \text { and } \quad S=2 \cdot \frac{I_{p}}{L} \cdot \frac{E}{2(1+v)}
$$

which for fixed $S_{0}$ leads to

$$
\frac{K}{S_{0}}=2 \cdot \frac{S_{0}^{2}}{E^{2}} \cdot \frac{I_{x}}{I_{p}^{3}} \cdot(1+v)^{3}
$$

where $I_{\mathrm{p}}$ and $I_{x}$ are the second moment of area for rotation and bending respectively, $E$ is the Youngs modulus, $L$ is the length of each of the two torsional beams and $v$ is Poisson's ratio. Eq. (1) clearly indicates that a large rotational compliance combined with a large vertical stiffness can only be obtained using compliant materials, i.e. with low Youngs modulus and appropriate beam-cross-sections. A nice reference to nature despite the fact that our torsional suspension and the cricket hairsockets have little in common. 


\section{F. Figure of Merit}

Optimization of our hair-sensors has been driven by a Figure of Merit (FoM) incorporating $L, d$ and $S$ and basically being the product of mechanical sensitivity and bandwidth [22]. This has emphasized what could be learned directly from observation of cricket hair-sensors, i.e. that hairs should be long, thin and mounted on very compliant suspensions. However, with respect to damping the optimum damping factors still need to be identified.

\section{HAIR-SENSOR ARRAYS}

Despite the marvelous performance of cricket single hairsensors [7] it seems to stand to reason that the overall function of the hair-sensors can only be fully appreciated when the hair-sensors are viewed in the context of their array configurations and their neural innervation patterns.

\section{A. Hair canopy as signal processor}

Without doubt the hair-canopy found on the cerci of crickets does not show an accidental pattern of hairs. It has been shown that hair-patterns and orientation are quite similar for crickets of the same species and instar [23]. Therefore it has been proposed that the cerci, in combination with the underlying neural apparatus, can perform specific filter functions [24]. For example, in [25] it is hypothesized how a combination of sequential mechanical excitation of the hairsensors in combination with a limited neural spike propagation velocity can lead to strong(er) signals when excited from the rear of the crickets than from the front. Hence this mechanism would work as a delay-line thereby strengthening the directionality of the cercal system and preferentially improve sensing from the rear where other sensory modalities are failing.

\section{B. Hair density and viscous coupling}

Inspired by the large densities of hairs found on crickets cerci, we studied viscous coupling between hair-sensors. I.e. the effect that one hair-sensor has on another neighboring hairsensor by virtue of the finite viscosity of air. For example when one hair sits upstream of another, one may expect that the boundary layer around the first hair will impact on the flow around the second hair in case this latter hair is near to the first one. As such viscous coupling poses limitations on the maximum density of hair-sensors that can be attained while still being useful. Moreover, since boundary layers around hairs are frequency dependent it stands to reason that viscous coupling effects will alter the frequency characteristics of hair-canopies relative to single hair-sensors. To identify these effects in more detail we have carried out a multiple facetted study $[26,27,28]$ on viscous coupling on cricket and artificial hair-sensors in the framework of the EU project CILIA [29] using previous analyses given in $[30,31,32]$ as a starting point.

In summary viscous coupling effects seem to merely scale with the relative inter-hair distance ( $r / d$ where $r$ is the distance between the hairs, $d$ the hair-diameter); the smaller this relative distance the stronger the effects, see Fig. 5. Of course also frequency plays a role since the boundary layer around the hairs is depending on it. As stated before this boundary layer reduces with increasing frequency and hence viscous

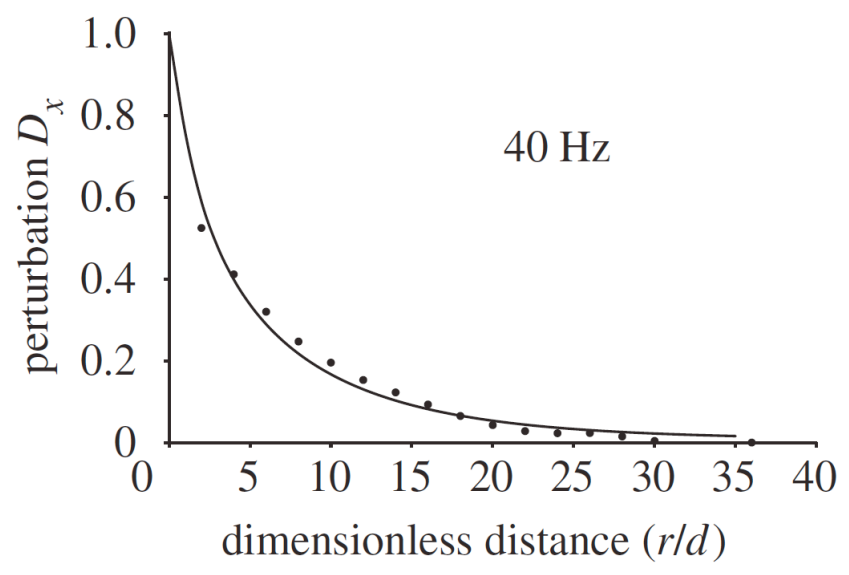

Figure 5: Extent of viscous coupling as a function of the normalized inter-hair distance as determined from MEMS hairs. Dots are measurements, the solid line theory [30]. Adapted from [26].

coupling effects become smaller as frequency increases. These effects were predicted in the analytic analysis given in [30], reproduced by numerical studies [27] and experimentally observed for fixed hairs in [26]. All this evidence has taught us that hair-spacings of less than 10 - 20 hair-diameters, as previously used in our arrays [33] with the intent to eventually make high density flow-observations, have profound influences on the flow-induced drag-torque acting on the hairs. Therefore next generation arrays will be fitted with lower hairdensity. This is a lesson that could be easily learned from studies of the cricket cerci as well since relative inter-hair distances seem to peak between 5 and 7 hair-diameters [34], as opposed to the $3-5$ hair-diameters of some of our previous designs [35].

\section{Hair-sensor orientation}

The hair-sensor orientation varies in a specific way over the cricket cerci [23] and it is believed that this aids crickets in an accurate directivity. However, how this is achieved can only be inferred when the neural "wiring" is fully understood, which is currently not the case. So it is expected that further studies of hair-orientation may give additional clues as to how to optimize the artificial sensor arrays.

\section{Hair-length variability}

Superficial inspection of cricket (and other arthropod's) hair canopies may suggest that the large variability of hair-length is related to frequency fractionation. However, the prevailing hair-air interaction models $[15,19]$ show that the hair-sensor response is well-damped, leading to rather flat frequency response curves which make frequency fractionation by hairlength variability unlikely. Therefore the question to the actual relevance of the hair-length variability is still not satisfyingly answered. A clue to the answer of this question may be in the hair-deflection response to transient stimuli as investigated in [2] and [20]. Without doubt, when this question has been solved it will give engineers valuable insights into further optimization of their hair-sensor arrays. 


\section{SUBSTRATE CONSIDERATIONS}

A marked difference between the cricket and artificial hair-sensor substrates is that the first is a rounded conical substrate whereas the latter is more conveniently chosen to be a flat substrate (silicon wafer). By investigation of the aerodynamics of the conical shape of cricket cerci we learned that such a shape improves sensitivity as far as the circumferential component of the flow is considered [36]. Therefore, both theoretically [37] as well as experimentally it turns out to be advantageous to mount hair-sensors on conical shapes. Alas, this is something not easily attainable to artificial MEMS-hairs although some examples have shown hair-sensors on non flatsubstrates $[38,39]$.

\section{DISCUSSION AND CONCLUSIONS}

On comparison of artificial and cricket hair-sensors one find that there are about 3 major difficulties for the previous in attaining comparable performance: a) rotational stiffness, b) thin hairs and c) sensitive, localized read-out.

\section{A. Rotational stiffness}

The problem with a simple torsional beam design has been described above. For longer, thinner beams the vertical stiffness $(K)$ becomes smaller such that the capacitor plates will easily pull-in onto the substrate on application of the voltages needed for the capacitive read-out. We are currently investigating both more advanced cross-sections (e.g. Tshaped beams) as well as materials with lower Youngs modulus (SU-8 and aluminum).

\section{B. Hair fabrication}

Fabrication of thin hairs is inherently limited by materials and deposition limitations. Where organisms can use DNA controlled assembly of molecules to virtually make any kind and any 3D shaped structure, MEMS technology is mostly based on photolithography that only allows for pseudo 3D fabrication. However, experimenting with less conventional exposure methods one may find ways to make more appropriate and nature-like hair-shapes [20].

\section{Sensor interfacing}

The beauty of the neural system lies in its robustness as well as its largely localized generation of signals. In contrast, the capacitive measurement method employed in our sensors is plagued by parasitic capacitances that can hardly be avoided and have a far more non-localized nature. E.g. changes in temperature, humidity, wiring geometry, etc. cause fluctuations of the parasitics which are large relative to the small capacitive changes that the hair-sensors induce. Therefore control of the parasitics is of paramount importance for a proper device functioning [40]. Nevertheless, sensor engineers can only envy the perfor-mance of the neural system.

\section{Current status}

In successive iterations we have been able to make airflow sensors, utilizing up to $1 \mathrm{~mm}$ long segmented cylinders mounted on rotatable membranes with electrodes allowing for differential capacitive read-out, delivering sensitivities of about $400 \mu \mathrm{m} / \mathrm{s}$ (300 Hz bandwidth) with near-perfect figure

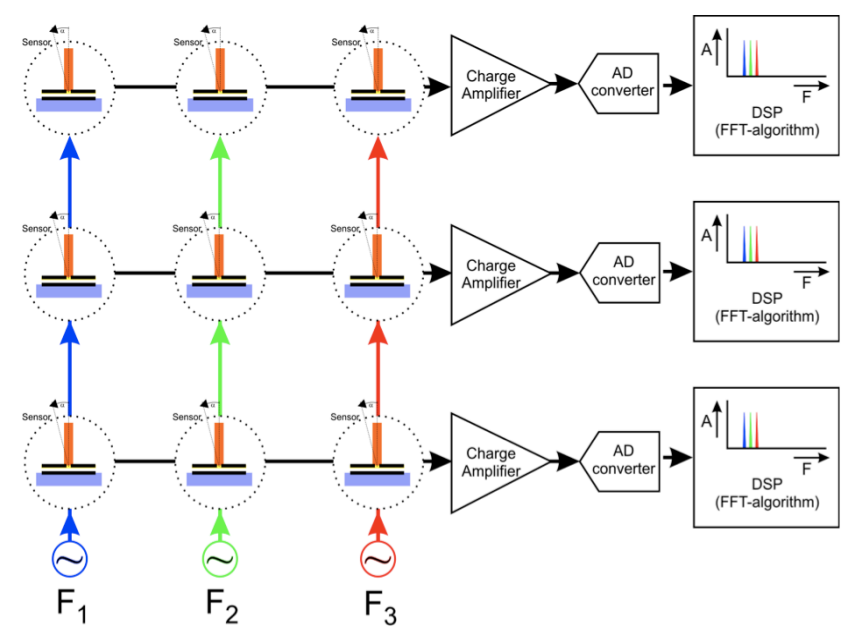

Figure 6: Schematic of Frequency Division Multiplexing as applied to hair-sensor array interacing [41].

of eight directivity. Moreover we can fabricate arrays of sensors (over 100) and utilizing Frequency Division Multiplexing these sensors can be simultaneously and individually interrogated (see Fig. 6) [41]. This allows us to carry out measurements of spatio-temporal flow-patterns rather than single point measurements. Hence we work towards a system delivering the functionality of a "flow-camera" with sensor densities of about $25-100$ sensors per $\mathrm{mm}^{2}$. This development has largely benefitted from insights in the cricket cercal system as explained in this contribution.

\section{ACKNOWLEDGMENT}

Without the many discussions we have had with our colleagues in the Cicada [42] and Cilia [29] projects this work would never have had the depth and breadth we have attained. We especially want to mention the fruitful collaboration with Jérôme Casas and his group at the Institut de Recherche sur la Biologie de l'Insecte (IRBI) at the Université François Rabelais, Tours, France. There have been many students, postdocs and technicians who contributed to the work reviewed in this paper. In alphabetical order these are: Dominique Altpeter, John van Baar, Erwin Berenschot, Rick de Boer, Meint de Boer, Christiaan Bruininck, Ahmad Dagamseh, Marcel Dijkstra, Harmen Droogendijk, Arjan Floris, Bjorn Hagendoorn, Nima Izadi, Marcel Kolster, Winfred Kuipers, Remco Sanders, Satya Shankar Siripurapu, Thomas Steinmann, Vitaly Svetovoy, Ramasubramanian Kottumakulal Jaganatharaja (a.k.a. Ram), Shrey Vats, Bas Verlaat and Henk van Wolferen.

\section{REFERENCES}

[1] H. Lamb, "The dynamical theory of sound", Edward Arnold, London, 1910.

[2] R. Kant and J. A. C. Humphrey, "Response of cricket and spider motion-sensing hairs to airflow pulsations", J. R. Soc. Interface, 2009, doi:10.1098/rsif.2008.0523

[3] Jürgen Tautz and Hubert Markl, "Caterpillars Detect Flying Wasps by Hairs Sensitive to Airborne Vibration”, Behav. Ecol. Sociobiol. 4, 101110 (1978). 
[4] J. Paton, R. Capranica, P. Dragsten and W. Webb, "Physical Basis for Auditory Frequency Analysis in Field Crickets (Gryllidae)", J. comp. Physiol. 119, 1977, p221-240.

[5] R. Sarpeshkar, "The silicon cochlea", Ch. 7 in Sensors and Sensing in Biology and Engineering, ed. Barth, Humphry and Secomb, Springer, Vienna, 2003, ISBN 3-211-83771-X.

[6] F.G. Barth, "Spider mechanosreceptors", Cur. Opinion in Neurobiology, Vol-14, 2004, page 415-422.

[7] Shimozawa, T. et. al., "Cricket wind receptors: thermal noise for the highest sensitivity known", Ch. 10, Sensors and Sensing in Biology and Engineering, ed. Barth, Humphry and Secomb, Springer, Vienna, 2003, ISBN 3-211-83771-X.

[8] M. Kanou, T. Osawa and T. Shimozawa, "Mechanical polarization in the air-current sensory hair of a cricket", Experientia 45, 1989, 1082 1083.

[9] W. Gnatzy and J. Tautz, Cell Tissue Res. 213 (1980) 441.

[10] F. Theunissen and J. Miller, "Representation of sensory information in the cricket cercal sensory system. II. Information theoretic calculation of system accuracy and optimal tuning curve widths of four primary interneurons", J. Neurophysiol. 66, 1991, 1690-1703.

[11] A. J. Kalmijn, "Hydrodynamic and Acoustic Field Detection," in Sensory biology of aquatic animals, J. Atema, et al., Eds., ed: SpringerVerlag, 1988, pp. 83-130.

[12] J. Franosch, M. Sobotka, A. Elepfandt, J. van Hemmen, "Minimal model of prey localization through the lateral-line system", Phys. Rev. Lett 91:158101-1/4.

[13] For MicroChem SU-8 data-sheets see http://www.microchem.com.

[14] Stokes, G.G. 1851 "On the effect of the internal friction of fluids on $\mathrm{t} \mathrm{h}$ e motion of pendulums", Transactions of the Cambridge Philosophical Society, Vol. IX. p. 8], reprinted in Mathematical and Physical Papers, vol. 111, pp. 1-141, Cambridge University Press, 1901.)

[15] J. Humphrey, R. Devarakonda, I. Iglesias, F. Barth, "Dynamics of arthropod filiform hairs. I. Mathematical modeling of the hair and air motions", Phil. Trans.: Bio. Sc. 340, 423-444, 1993.

[16] M. Dijkstra, J. van Baar, R. Wiegerink, T. Lammerink, J. de Boer and G. Krijnen, "Artificial sensory hairs based on the flow sensitive receptor hairs of crickets", J. Microm. Microeng. 15, S132-S138, 2005.

[17] R. Jaganatharaja, C. Bruinink, B. Hagedoorn, M. Kolster, T. Lammerink, R. Wiegerink and G. Krijnen, "Highly-sensitive, biomimetic hair sensor arrays for sensing low-frequency air flows", proc. Transducers 2009, Denver, USA, 21 - 25 June 2009, pg 1541 1544.

[18] M. McConney, C. Schaber, M. Julian, F. Barth, V. Tsukruk, "Viscoelastic nanoscale properties of cuticle contribute to the high-pass properties of spider vibration receptor (Cupiennius salei Keys)", J. Royal Soc. Interface ,Vol 4, 2007, p1135-1143, doi: 10.1098/rsif.2007.1000

[19] T. Shimozawa, T. Kumagai, Y. Baba, "Structural scaling and functional design of the cercal wind-receptor hair of cricket", J. Comp. Physiology A 183,1998, p171-186.

[20] H. Droogendijk et. al, in preparation.

[21] R. Kottumakulal Jaganatharaja et. al, in preparation.

[22] G. Krijnen, T. Lammerink, R. Wiegerink and Jérôme Casas, "Cricket Inspired Flow-Sensor Arrays", Proc. IEEE Sensors 2007, Atlanta, p539 $-546$.

[23] J. Palka, R. Levine and M. Schubiger, "The Cercus-to-Giant Interneuron System of Crickets I. Some Attributes of the Sensory Cells", J. comp. Physiol. 119, 1977, p267-283.
[24] G. Jacobs, J. Miller and Z. Aldworth, "Computational mechanisms of mechanosensory processing in the cricket", J. Exp. Biology 211, 2010, p1819 - 1828, doi: 10.1242/jeb.016402.

[25] J. Mulder-Rosi, G. Cummins, J. Miller, "The cricket cercal system implements delay-line processing" J. Neurophysiol. In press. doi: 10.1152/jn.00875.2009.

[26] J. Casas, T. Steinmann and G. Krijnen, "Why do insects have such a high density of flow-sensing hairs? Insights from the hydromechanics of biomimetic MEMS sensors", J. Royal Soc. Interface, in press doi: 10.1098/rsif.2010.0093.

[27] G. C. Lewin and J. Hallam, "A computational fluid dynamics model of viscous coupling of hairs", J Comp Physiol A, in press, doi: 10.1007/s00359-010-0524-6

[28] B. Hagendoorn, "Viscous Coupling Between Bio-Inspired HairSensors", MSc. thesis, University of Twente, April, 2009.

[29] Customized Intelligent Life Inspired Arrays, Cilia: http://www.bionics-cilia.org/.

[30] B. Bathellier, F. Barth, J. Albert and J. Humphrey, "Viscosity-mediated motion coupling between pairs of trichobothria on the leg of the spider Cupiennius salei", J. Comp. Physiol. A 191, 2005, p733-746. [Err. in J. Comp. Physiol. A 2010 196, 89.] (doi: 10.1007/s00359-005-0629-5).

[31] B. Cummins, T. Gedeon, I. Klapper and R. Cortez, "Interaction between arthropod filiform hairs in a fluid environment", J. Theor. Biol. 247, 266-280. (doi:10.1016/j.jtbi.2007.02.003).

[32] J. Heys, T. Gedeon, B. Knott and Y. Kim, "Modeling arthropod filiform hair motion using the penalty immersed boundary method. J. Biomech. 41, 977-984. (doi: 10.1016/j.jbiomech.2007.12.015).

[33] G. Krijnen, et al., "MEMS based hair flow-sensors as model systems for acoustic perception studies", Nanotechnology, vol 17, pp. 84-89, 2006.

[34] O. Dangles, T. Steinmann, D. Pierre, F. Vannier and J. Casas, "Relative contributions of organ shape and receptor arrangement to the design of cricket's cercal system", J Comp Physiol A, Vol 194, 2008, p653-663, doi: 10.1007/s00359-008-0339-x

[35] J. Floris, N. Izadi, R. Jaganatharaja, R. Wiegerink, T. Lammerink and G. Krijnen, "Adaptation for frequency focusing and increased sensitivity in biomimetic flow sensors using electrostatic spring softening", proc. Tranducers 2007, Lyon, France, paper 2EO4.P, p1267 - 1270.

[36] T. Steinmann, J. Casas, G. Krijnen and O. Dangles, “Air-flow sensitive hairs: boundary layers in oscillatory flows around arthropod appendages” J. Exp. Bio. vol-209, 4398-4408, 2006.

[37] J. Holtsmark, I. Johnsen, T. Sikkeland and S. Skavlem, "Boundary layer flow near a cylindrical obstacle in oscillating, incompressible fluid", J Acoust Soc Am, Vol 26, 1954, p26 - 39.

[38] J Engel, J. Chen, C. Liu and D.Bullen, "Polyurethane Rubber AllPolymer Artificial Hair Cell Sensor". J. Microelectromechanical Sys., Vol. 15, August 2006, p729 - 736.

[39] G. Krijnen, A. Floris, M. Dijkstra, T. Lammerink, R. Wiegerink, "Biomimetic micromechanical adaptive flow-sensor arrays", SPIE Proc. vol. 6592, paper 6592-16, 2007.

[40] A. Dagamseh, C. Bruinink, H. Droogendijk, R. Wiegerink, T. Lammerink and G. Krijnen, "Engineering of Biomimetic Hair-Flow Sensor Arrays Dedicated to High-Resolution Flow Field Measurements", proc. IEEE Sensors 2010, Waikola, this proceeding.

[41] A. Dagamseh, C. Bruinink, M. Kolster, R. Wiegerink, T. Lammerink and G. Krijnen, "Array of biomimetic hair sensor dedicated for flowpattern recognition", proc. DTIP 2010, 5-7 May 2010, Seville, Spain, ISBN: 978-2-35500-011-9.

[42] CrIcket perCeption And Decision Automata, Cicada: http://www.bionics-cicada.org 